\title{
Obstetric Safety Patient
}

\author{
Antonio Ragusa, Shin Ushiro, Alessandro Svelato, \\ Noemi Strambi, and Mariarosaria Di Tommaso
}

\subsection{Introduction}

In healthcare, the patient safety system which has been developed following the study of the various phases necessary for its determination, supplies strategies to avoid the repetition of circumstances that originally has led an individual to make mistakes. In fact, the culture of risk management, starting from the consideration that the errors are not eliminable, is based on the belief that they need to be properly analyzed, implementing intervention strategies that avoid its repetition, in order to become good learning opportunities.

The risk is the condition or potential event, intrinsic or extrinsic to the process, which can modify the expected outcome. It is measured in terms of probability and consequences, as a prod-

A. Ragusa $(\bowtie) \cdot$ A. Svelato

Obstetrics and Gynecology Department,

Fatebenefratelli Hospital, San Giovanni Calibita,

Rome, Italy

\section{S. Ushiro}

Division of Patient Safety, Kyushu University

Hospital, Fukuoka, Japan

e-mail: ushiro@surg2.med.kyushu-u.ac.jp

\section{N. Strambi · M. Di Tommaso}

Department of Health Sciences, Obstetrics and Gynecology, University of Florence, Careggi Hospital Florence, Florence, Italy

Fetal Maternal Department, AOU Careggi Hospital, Florence, Italy

e-mail: mariarosaria.ditommaso@unifi.it uct of the probability that a specific event can occur and the seriousness of the damage that can follow this. In the calculation of risk, human ability to identify and contain the consequences of the potentially harmful event is also considered [1].

In obstetrics, there is cultural confusion regarding the concept of risk, as a measurement of the probability of damage in a given population, and the concept of risk as the presence of danger for an individual.

That is, the approach which considers all women to be at risk, without systematically defining the degree of probability with which a complication can occur in a specifically assisted obstetric condition, is not functional, nor positive regarding assistance for several reasons:

- Cultural: the culture of risk increases the anxiety of operators and women. While scientific investigation of uncertainty promises to increase safety; in actual fact, it increases our insecurity, distorting the emphasis on the prevention of impending risks, and transforming the majority of healthy pregnant women into pre-sick people.

- Organizational: no healthcare system can always guarantee maximum efficiency. It is useful that the organization is optimized for a significant event, while it is reasonable to apply low intensity assistance in the normal course of activity. 
- Pragmatic: in a healthcare system, operators cannot be kept on continuous alert so as not to let down their guard precisely in the circumstances in which they should be fully present.

- Epidemiological: the value of a diagnostic test useful to highlight the presence of a maternal or fetal pathology depends on the knowledge of the a priori probability that pathology occurs in the population under investigation.

For Bayes' theorem, the positive predictive value of a diagnostic test is directly related to the prevalence of the negative outcome, to be avoided in the population under investigation [2]. In other words, the presence of a pathological test is really indicative of pathology, the greater the a priori prevalence of the same in the subject undergoing the test. Vice versa, the percentage of false positives of the test is greater the lower the probability of the appearance of pathology in the subject undergoing the test.

If the prevalence is not taken into account, there will be a much more frequent alarm than necessary. For example, in a physiological population with low probability $(<1 \%)$ of having a fetal acidosis, the presence of a pathological cardiotocographic tracing implies an effective presence of acidosis in $16 \%$ of cases. Vice versa, in a pre-eclamptic patient, who has a high probability of an acidosis risk (about $30 \%$ ), due to placental hypoperfusion and the consequently reduced reserves in the underdeveloped fetus, the same tracing implies the presence of acidosis in $89 \%$ of cases. From these considerations it follows that it is appropriate to have a greater or lesser diligence or interventionism in the presence of pathological traces in physiology and risk.

A rational attitude is therefore that of getting used to mentally cataloging the a priori probability of possible pathologies, which the woman or fetus that is seen during pregnancy or in labor could present. Although it is not possible to foresee everything, it is necessary to be aware that a pathology is not equally distributed in all pregnancies, but will depend on specific variables that we must take into account every time we take care of a woman, to optimize assistance in a per- sonalized way [3]. In conclusion, it is not possible to define a woman at risk without defining the type of risk and the probability that this risk can develop.

A further problem directly related to the safety of the obstetric patient derives from the fact that in obstetrics, perhaps more than in other medical disciplines, we can witness a rapid transition from a situation of well-being to an acute pathology, moreover in a context such as birth that is usually accompanied by positive, celebratory emotions. This immediately leads both operators and families to think that something has not been done as it should have been [4].

But it is a fact that adverse events are ubiquitous in today's clinical practices despite the best intentions to improve patient health. If complications related to the course of a certain disease or specific treatment have been accepted for centuries as part of the care process; another matter is the question of error. It is only since the end of the last century that we have started talking about the prevention of adverse events or claims for damages related to an error. Human and systemic errors are intrinsic to the complex care system and we are well aware of their weight in medicine. So this is why all possible strategies must be put in place to avoid a foreseeable error through risk management. Getting and making things right when things go wrong defines a successful safety program [5].

\subsection{Patient Safety}

Risk management identifies a set of actions which improve the quality of health services in order to guarantee patient safety. Risk management tools are represented by four processes: identification, analysis, control, and financial coverage of risk.

Risk investigation is the process by which situations, the user and the procedure are identified, which can lead, or have led, to a loss. The approach is based on the assumption that any error is the consequence of problems that precede it and that such problems could become manifest even before the adverse event occurs. 
The map of critical areas identifies various criticalities in different ways; it presupposes the presence of a surveillance epidemiological observatory and can be carried out according to the needs of the research, the time, the concentration of adverse events in a given sector, the severity of the adverse events, etc. Its interpretation must always be very cautious as a starting point for a critical analysis and not used as the conclusive outcome of an investigation.

Risk control consists of the implementation of prevention procedures and strategies that lead to the creation of a specific risk prevention/mitigation plan. The control focuses on the training of employees in terms of information, consent, accurate compilation of a medical record, hospital discharge sheets, and reporting of unwanted events. It should also concentrate on the development of protocols, procedures, and/or control measures that can improve the safety of the assisted person and on the efficiency of the risk management units understood as monitoring capacity, interpretation of the causes of unwanted events, and identification of clinical corrective factors.

In the context of control measures, particular importance is given to the audit which is a formal process of clinical verification that controls the effectiveness of the interventions while evaluating the assistance in its various components. It aims to improve the quality and outcomes of patient care through a structured review conducted by groups of colleagues, that is with peer reviews, which after examining the clinical practice used and its results, based on the standards adopted and the elements that emerge from the verification, provide any necessary indications to modify it. The audit must give answers to questions concerning the service provided to the patient by all the professionals involved. Financial risk coverage must identify the funds necessary to cover the risk management plan and must necessarily also include insurance coverage of the settings most exposed to financial risk.

Integrated management must involve changes in clinical practice aimed at promoting a culture of safety that is more attentive and closer to the dual patient mother/fetus and infant, as well as to operators. Therefore, in the first instance it is useful to identify the training, organizational and technological criticalities encountered in the maternal-infant clinical path with reference to pregnancy, childbirth, and assistance to the newborn. The training criticalities are also to be considered in relation to the reduced volume of activities, while the organizational ones are mostly linked to a lack of continuity in the territory/hospital care and due to the lack of neonatal intensive care beds. A recent review also suggests that educational interventions aimed at improving the quality of care and training health workers may improve the safety of women and their infants during childbirth [6]. In the second instance, all risk management actions must be reported through the prevention of their realization, so as to constitute a sort of "risk control plan in the maternal and child area" [7].

In the maternal and child care area, risk management must involve all sectors in which an error can materialize in various phases of the mother and the newborn to be effective. In terms of obstetrics, attention to the three types of criticality: training, organization, and technology must focus on prevention in the preconception phase, during pregnancy and during childbirth assistance.

The clinical assistance to pregnancy and labor begins in the preconception period because it is an important time concerning the prevention of some risks, which should be identified and corrected before the concretization of these risks. The main risks are malformations, genetic, teratogenic caused by physical and toxic agents, infectious, deficiency, coming from maternal-fetal incompatibility and prematurity. In this phase, the anamnesis plays an important role in the identification of the risk, and criticalities that can emerge are of exclusive pertinence training. Folic acid supplementation, the abolition of incorrect lifestyle habits (e.g., drugs and alcohol use, smoking), close glycemic control of diabetic women, lengthening the interval between pregnancies, are just some of the examples of malformation and prematurity risk containment already in the preconception phase. 
Risks associated with pregravidical anamnestic factors involved right from the start of pregnancy, especially if they have not already been carried out in the preconception period, must be identified, along with a timely diagnosis of extrauterine pregnancy. The speed here avoids, first of all, the need to intervene in emergency situations due to serious hypovolemic maternal shock related to hemopertoneal as a consequence of extrauterine pregnancy rupture, and secondly, it allows for more conservative treatments and less invasive interventions, such as medical treatment with methotrexate and video laparoscopy of the tubes. In this context, the critical points that can be detected often concern training aspects. The use of a sort of checklist aimed at identifying anamnestic risk factors important for pregnancy management right from the beginning of a pregnancy can be of great help, and it is the first step of the obstetric triage whose task is to highlight specific care pathways for the assessment of the risk profile which is a dynamic concept in continuous evolution during pregnancy. The minimal number of maternal screening tests to be carried out in the antenatal period which must be guaranteed to every woman are identified by the Maternity Clinical Risk Management Standards (CNST) [8]. The fetal screening involves fetal anomalies and Down Syndrome, the maternal screening involves infection in pregnancy such as rubella, hepatitis B, HIV, syphilis, and hemoglobinopathies such as sickle cell anemia and thalassemia [9].

During pregnancy, the correct surveillance of pregravidic diseases such as heart disease, respiratory failure, and hypertension is essential in order to monitor their possible deterioration, for the protection of maternal health and life. To safeguard the health of the future newborn, the timely diagnosis of fetal growth restriction and the realization of the risk of spontaneous premature birth allows optimization of the survival and quality of life of "small" infants both by optimizing the timing of birth in the case of fetal growth restriction, and by centralizing pregnant women (transport in utero) to hospitals equipped with neonatal intensive care whenever a premature baby is expected to be born.
Childbirth labor remains, however, the most critical phase for the safeguarding of the health and life of women. Obstetric emergencies such as postpartum hemorrhage, eclampsia, sepsis, thromboembolism, and anesthetic intervention are clinical aspects that must be monitored to prevent maternal death or serious disease related to labor and delivery.

During labor, cord prolapse, uterine rupture, uterine inversion, and shoulder dystocia are among obstetric emergencies that require timely and adequate treatments that not only require specific protocols, but also a pre-ordered and routinely monitored organizational support network using checklists, simulations, "mnemonic" and "reminder" posters.

Even vaginal delivery after a cesarean section, operative delivery and the so-called cardiotocographic emergencies are clinical pathway events that deviate from physiology, but since they are part of obstetric pathology they must, in any case, be foreseen. For each of these occurrences the risk factors must be identified a priori, as they are often, but not always present, for their realization and the treatment plans to be implemented [10].

In the clinical path, the analysis of errors represents an effective tool for prevention through the construction of barriers that prevent the realization of the damage that can result. In the obstetric area, as well, efforts have been and are still being made in order to identify errors and causes of mortality and morbidity in advance in order to offer safety indicators [11].

\subsection{Most Frequent Errors and Adverse Events}

The creation of these barriers is facilitated by the identification of missed missions, the so-called near-misses, defined as unscheduled events caused by errors that, however, do not determine the damage that they were potentially able to achieve. Through identification and analysis of a system of errors that create damage and nearmiss events in the labor and delivery room, it is possible to identify interventions to reduce potential damage. The first systematic review of 
near-miss events in obstetrics is recent and reports an incidence of $0.69 \%$, but refers to a context with a careful multi-year organization on patient safety and, as such, the data cannot be generalized. In that context, the analysis of unexpected events, including near-misses, in the labor-delivery room was 3959 cases in 2010 out of a total of 203,708 births, with an incidence of $1.9 \%$. For each near-miss event, the hazard score is reported based on four parameters: (a) the worst possible outcome; (b) the identification method; (c) the number of barriers encountered; and (d) the quality of the barriers. Through the hazard score, it is possible to outline four classes of events: (1) high-risk frequency and low hazard score; (2) high frequency and high hazard score; (3) low-risk frequency and high hazard score; (4) low-risk frequency and low hazard score [12].

Since interventions based on the use of checklists integrated into clinical practice have proven to be effective in reducing death and complications both in the area of intensive care and surgery, the World Health Organization for Developing Countries has developed a checklist, the WHO Safe Childbirth Checklist, which focuses on the major causes of morbidity and mortality in the mother and newborn during delivery [13]. In fact, patient safety has a measurable economic effect, saves lives, and reduces morbidity. The reduction in mortality associated with birth is a priority of global health and low quality care is recognized to be the factor that most contributes to birth-related harm.

A systematic analysis describes global levels and trends in maternal mortality between 1990 and 2015. The global maternal mortality ratio (MMR) has a relative decline of $43.9 \%$ (34.0 48.7) from 1990 to 2015. The MMR reduced 385 deaths per 100,000 live births (80\% uncertainty interval ranges from 359 to 427) in 1990 to 216 deaths (207-249) in 2015 with 303,000 (291,000 $349,000)$ maternal deaths globally. Even though there is a global decrease of maternal mortality, this progress should be accelerated and immediate action is necessary to substantially reduce preventable maternal deaths [14]. The percentage of maternal deaths that can be foreseen and there- fore preventable varies from $28 \%$ to $50 \%$ [15-17].

In order to introduce the patient safety concept to those making decisions especially in poor resources settings, the WHO Safe Childbirth Checklist guides the selection of patient safety policy points to ensure that the national policy is comprehensive and adequately detailed.

The items on this document identify the major causes of maternal and neonatal death in developing countries. Examples include postpartum hemorrhage, dystocic labur, hypertensive disorders, intrapartum events such as suboptimal assistance to the mother, neonatal infections, and prematurity. The items are grouped to be used in four critical and crucial moments: (a) on admission of the woman to the hospital; (b) at the beginning of the expulsion period or before the cesarean section; (c) $1 \mathrm{~h}$ after birth and (d) before discharge [18].

The pilot study of implementation of the checklist showed a clear improvement in terms of maternal-fetal neonatal health which makes it very promising. For the preparation of checklists, the priority identification of recurrent errors is useful, as only by recognizing them can they be avoided. Table 16.1 shows in descending order, the most common and frequent errors for some obstetric emergencies, highlighted during simulations [19].

Another risk control tool in obstetrics is that of the MEOWS (Modified Early Obstetric Warning System), an early alarm system for the timely recognition and treatment of all acute pathological situations, developed on the basis of the Confidential Enquiry into Maternal and Child Health report of 2003-2005, the validation of which has proved to be a useful aid to be used at the patient's bedside to predict morbidity, whose diagnostic criteria and alarm threshold parameters, and "trigger points," are well defined [20, 21] and reported in Table 16.2 [22].

Furthermore, this system can be easily learned, implemented, and interpreted. Available evidence suggests that MEOWS should enhance surveillance programs and action plans in order to reduce severe maternal morbidity and mortality 
Table 16.1 Common and Recurrent Errors Detected by Simulation

\begin{tabular}{|c|c|}
\hline Scenario & Error \\
\hline $\begin{array}{l}\text { Eclamptic } \\
\text { seizure }\end{array}$ & $\begin{array}{l}\text { Inappropriate ventilation technique } \\
\text { Incorrect treatment of } \mathrm{MgSO}_{4} \\
\text { intoxication } \\
\text { Underdetection of } \mathrm{MgSO}_{4} \\
\text { intoxication } \\
\text { No ventilation performed in an } \\
\text { apneic patient } \\
\text { Essential blood tests (liver and renal } \\
\text { function, coagulation) not carried out } \\
\text { Unfamiliarity with hydralazine } \\
\text { dosage } \\
\text { Incorrect dosage or dilution of } \\
\text { MgSO}_{4} \\
\text { Foley catheter not inserted }\end{array}$ \\
\hline $\begin{array}{l}\text { Postpartum } \\
\text { hemorrhage }\end{array}$ & $\begin{array}{l}\text { Underestimation of blood loss } \\
\text { Unfamiliarity with prostaglandin } \\
\text { administration to achieve myometrial } \\
\text { contraction } \\
\text { Late transition to the operating room } \\
\text { Delayed administration of blood } \\
\text { products } \\
\text { Unfamiliarity with ergot myometrial } \\
\text { stimulators dosage } \\
\text { Essentials blood test (fibrinogen, PT, } \\
\text { PTT, cross and match) not carried } \\
\text { out } \\
\text { Under detection of consumption } \\
\text { coagulopathy } \\
\text { Source of bleeding (episiotomy } \\
\text { wound exploration, uterine cavity } \\
\text { revision, etc.) not explored } \\
\text { Urinary bladder not drained }\end{array}$ \\
\hline $\begin{array}{l}\text { Shoulder } \\
\text { dystocia drill }\end{array}$ & $\begin{array}{l}\text { Inadequate documentation of the } \\
\text { event } \\
\text { Delayed episiotomy } \\
\text { Ineffective suprapubic pressure } \\
\text { Incorrect McRoberts technique } \\
\text { No episiotomy performed } \\
\text { Incorrect order of actions and } \\
\text { maneuvers }\end{array}$ \\
\hline $\begin{array}{l}\text { Breech } \\
\text { delivery }\end{array}$ & $\begin{array}{l}\text { Incorrect fixation of the limbs } \\
\text { Hasty attempt to deliver the arms } \\
\text { Inappropriate Mauriceau and Bracht } \\
\text { maneuvers } \\
\text { No episiotomy performed }\end{array}$ \\
\hline
\end{tabular}

by identifying, managing, and possibly avoiding preventable maternal adverse events [23].

A further element on which to base prevention is communication both between operators and with women and their relatives, focusing on information useful for current assistance.
Table 16.2 Limits of trigger thresholds for MEOWS parameters

\begin{tabular}{l|l|l}
\hline & Yellow trigger & Red trigger \\
\hline Temperature $\left({ }^{\circ} \mathrm{C}\right)$ & $35-36$ & $<35$ or $>38$ \\
\hline Systolic BP $(\mathrm{mmHg})$ & $\begin{array}{l}150-160 \text { or } \\
90-100\end{array}$ & $<90$ or $>160$ \\
\hline $\begin{array}{l}\text { Diastolic BP } \\
(\mathrm{mmHg})\end{array}$ & $90-100$ & $>100$ \\
\hline $\begin{array}{l}\text { Heart rate (beat/min) } \\
100-120 \text { or } \\
40-50\end{array}$ & $<40$ or $>120$ \\
\hline $\begin{array}{l}\text { Respiratory rate } \\
\text { (breaths/min) }\end{array}$ & $21-30$ & $<10$ or $>30$ \\
\hline $\begin{array}{l}\text { Oxygen saturation } \\
(\%)\end{array}$ & - & $<95$ \\
\hline Pain score & $2-3$ & $\begin{array}{l}\text { Unresponsive, } \\
\text { pain }\end{array}$ \\
\hline $\begin{array}{l}\text { Neurological } \\
\text { response }\end{array}$ & Voice & \\
\hline
\end{tabular}

In 2005, the Royal College of Obstetricians and Gynaecologists declared: "Essential elements of providing a good standard of practice and care are professional competence, good relationships and communication with patients and colleagues and observance of professional ethical obligations" [24]. Clear communication is synonymous with resolutive and cognitive skills, it also implies an accurate transcription of the event in the medical record and can mitigate the fallout of negative outcomes [25].

When an adverse event occurs, closing the circle with the patient is an essential component in doing things correctly. Many times, it is difficult to admit the incident, but the establishment of a relationship of trust at the basis of the doctor-patient relationship allows a better outcome also in medico-legal terms. Given the difficulty in communicating bad news, many different strategies have been develop regarding the disclosure method. For example, a real protocol has been developed at Yale University where communication with the patient is structured like all the various care processes [4, 26-28].

\subsection{Recommendation}

The cornerstones on which control and risk management in obstetrics is based are: 
(a) obstetric triage for the identification of protocols and specific care paths.

(b) the application of guidelines and protocols for different clinical situations related to the birth path.

(c) the use of checklists to analyze the most vulnerable points of the process accurately.

(d) simulations.

(e) the collection of data in delivery rooms must be precise and systematic, if the data collected will not be truthful or will be collected and described incorrectly, all the conclusions and consequent corrective measures will be wrong [26].

Additional elements on which accident prevention is based are: optimization of internal and external communication, communication with the patient, organization of the team and clinical documentation. However, intervening in these areas does not represent a specificity of the maternal infantile path but that of the prevention of adverse events in all medical-surgical disciplines.

In conclusion, the resulting safety system, developed following the study of the various phases of error determinism in three areas (training, organizational and technological), allows the implementation of risk control strategies that avoid the repetition of circumstances that lead to mistakes. The set of actions identified constitute a sort of risk control plan in the maternal and child area which, by improving the quality of the services provided to the mother and the newborn, should guarantee their safety [27].

Safeguarding the health and life of the mother and the future baby are the main objectives of a correct clinical course of pregnancy [28].

\section{References}

1. Leape LL. Reporting of adverse events. N Engl J Med. 2002;347(20):1633-8.

2. Godlee F. Are we at risk of being at risk? BMJ. 2010;341:c4766.

3. NHS National Reporting and Learning System Feedback Report. Safety first: a report for patients, clinicians and healthcare managers. 2006.
4. Pettker CM. Systematic approaches to adverse events in obstetrics, part II: event analysis and response. Semin Perinatol. 2017;41(3):156-60.

5. Pettker CM. Systematic approaches to adverse events in obstetrics, part I: event identification and classification. Semin Perinatol. 2017;41:151-5.

6. Antony J, Zarin W, Pham B, et al. Patient safety initiatives in obstetrics: a rapid review. BMJ Open. 2018;8:e020170.

7. RCOG, RCM, RCA, RCPCH. Safer childbirth. Minimum standards for the organisation and delivery of care in labour. London: RCOG Press; 2007. p. $1-80$.

8. https://resolution.nhs.uk/services/claims-management/ clinical-schemes/clinical-negligence-scheme-for-trusts/ maternity-incentive-scheme/.

9. NHS Litigation Authority. Clinical negligence scheme for trusts maternity clinical risk management standards 2012-13. Managing risk DNV. 2012.

10. ACOG Committee Opinion. Patient safety in obstetrics and gynecology, no. 447. 2009, reaffirmed 2019.

11. Pronovost PJ, Holzmuller CG, Ennen CS, Fox HE. Overview of progress in patient safety. Am J Obstet Gynecol. 2011;204:5-10.

12. Clark SL, Meyers RN, Frye DR, et al. A systematic approach to the identification and classification of near-miss events on labor and delivery in a large national health care system. Am J Obstet Gynecol. 2012;207:441-5.

13. WHO Safe Childbirth Checklist: implementation guide improving the quality of facility-based delivery for mothers and newborns. WHO Library Cataloguing. 2015.

14. Alkema L, ChouD, HoganD, etal. Global, regional, and national levels and trends in maternal mortality between 1990 and 2015, with scenario-based projections to 2030: a systematic analysis by the UN Maternal Mortality Estimation Inter-Agency Group. Lancet. 2016;387(10017):462-474.

15. Berg CJ, Harper MA, Atkinson SM, et al. Preventability of pregnancy-related deaths: results of a state-wide review. Obstet Gynecol. 2005;106:1228-34.

16. Clark SL, Belfort M, Dildy GA et al Maternal death in the 21st century: causes, prevention and relationship to cesarean delivery. Am J Obstet Gynecol. 2008;199(1):36e1-36e5.

17. Spector JM, Agrawal P, Kodkany B, et al. Improving quality of care for maternal and newborn health: prospective pilot study of the WHO Safe Childbirth Checklist Program. PLoS One. 2012;7(5):e35151.

18. Philip Banfield P, Roberts C. The early detection of maternal deterioration in pregnancy. London: The Health Foundation; 2015.

19. Maslovitz S, Barkai G, Lessing JB, et al. Recurrent obstetric management mistakes identified by simulation. Obstet Gynecol. 2007;109:1295-300.

20. Zuckerwise LC, Lipkind HS. Maternal early warning systems-towards reducing preventable maternal mortality and severe maternal morbidity through 
improved clinical surveillance and responsiveness. Semin Perinatol. 2017;41(3):161-5.

21. Wu AW, Steckelberg RC. Medical error, incident investigation and the second victim: doing better but feeling worse? BMJ Qual Saf. 2012;21(4):267-70.

22. Singh S, McGlennan A, England A, Simons R. A validation study of the CEMACH recommended modified early obstetric warning system (MEOWS). Anaesthesia. 2012;67:12-8.

23. Mackintosh N, Watson K, Rance S, Sandall J. Value of a modified early obstetric warning system (MEOWS) in managing maternal complications in the peripartum period: an ethnographic study. BMJ Qual Saf. 2014;23(1):26-34.

24. RCOG, Clinical Governance Advice. Improving patient safety: Risk management for maternity and gynaecology, no. 2. 2005.
25. Ragusa A, Crescini C. Emergenze Urgenze in sala parto, Piccin. 2015.

26. Svelato A, Ragusa A, Manfredi P. General methods for measuring and comparing medical interventions in childbirth: a framework. BMC Pregn Childbirth. 2020 (in press).

27. Pettker CM, Thung SF, Lipkind HS, et al. A comprehensive obstetric patient safety program reduces liability claims and payments. Am J Obstet Gynecol. 2014;211:319-25.

28. Gazzetta Ufficiale 131 del 7 giugno 2000. Decreto Ministeriale del 24.4.2000. Piano Sanitario Nazionale 1998-2000. Progetto Obiettivo Materno Infantile (POMI).

Open Access This chapter is licensed under the terms of the Creative Commons Attribution 4.0 International License (http://creativecommons.org/licenses/by/4.0/), which permits use, sharing, adaptation, distribution and reproduction in any medium or format, as long as you give appropriate credit to the original author(s) and the source, provide a link to the Creative Commons license and indicate if changes were made.

The images or other third party material in this chapter are included in the chapter's Creative Commons license, unless indicated otherwise in a credit line to the material. If material is not included in the chapter's Creative Commons license and your intended use is not permitted by statutory regulation or exceeds the permitted use, you will need to obtain permission directly from the copyright holder. 